\title{
Observations of the anisotropy of cosmic rays at $\mathrm{TeV}-\mathrm{PeV}$
}

\author{
S. BenZvi \\ Wisconsin IceCube Particle Astrophysics Center and Department of Physics, University of Wisconsin-Madison, \\ Madison, WI, USA \\ Correspondence to: S. BenZvi (sybenzvi@icecube.wisc.edu)
}

Received: 27 February 2014 - Revised: 26 May 2014 - Accepted: 3 June 2014 - Published: 25 July 2014

\begin{abstract}
During the past decade, multiple observatories have reported significant observations of the anisotropy of cosmic rays in the $\mathrm{TeV}$ energy band. The anisotropy has been observed at large scales and small scales in both the Northern and Southern Hemispheres. The source of the anisotropy is not well-understood, though both a galactic and a heliospheric origin have been suggested. We discuss recent observations of the shape and energy dependence of the anisotropy, with particular attention to measurements by the IceCube Neutrino Observatory in the Southern Hemisphere and the Milagro and High-Altitude Water Cherenkov (HAWC) observatories in the Northern Hemisphere.
\end{abstract}

\section{Introduction}

Recent observations of the distribution of arrival directions of $\mathrm{TeV}$ cosmic rays at Earth have demonstrated the presence of an anisotropy at an intensity level of $10^{-3}$. Measurements in the Northern Hemisphere were performed by the Tibet AS $\gamma$ array (Amenomori et al., 2005), Super-Kamiokande (Guillian et al., 2007), Milagro (Abdo et al., 2008, 2009), EAS-TOP (Aglietta et al., 2009), MINOS (de Jong, 2011), ARGO-YBJ (Di Sciascio, 2013; Bartoli et al., 2013), and most recently HAWC (BenZvi et al., 2013). In the southern sky observations covering a large energy range have been made with the IceCube (Abbasi et al., 2010, 2011, 2012) and IceTop (Aartsen et al., 2013) detectors.

The anisotropy has been observed on both large angular scales $\left(>60^{\circ}\right.$ in extent) and small scales $\left(<20^{\circ}\right)$ over the full sky. Including all observations performed during the past decade, the intensity of the large-scale anisotropy has been investigated between $1 \mathrm{TeV}$ and $1 \mathrm{PeV}$, and the time dependence of the large-scale structure has been observed continuously over the most recent solar cycle. We discuss these results in Sects. 2.1 and 2.2. The small-scale anisotropy is described in Sect. 3. Although the source of the anisotropy is not well-understood, we describe several origin scenarios as the data are presented.

\section{Large-scale structure}

The large-scale anisotropy in the $\mathrm{TeV}$ cosmic rays can be described as a linear combination of dipole and quadrupole components. Defining the relative intensity of the distribution of cosmic ray arrival directions as

$\delta I(\alpha, \delta)=\frac{\Delta N}{\langle N\rangle}=\frac{N(\alpha, \delta)-\langle N(\alpha, \delta)\rangle}{\langle N(\alpha, \delta)\rangle}$,

where $\alpha$ and $\delta$ are right ascension and declination, and $N$ is the number of events at $(\alpha, \delta)$, we find that the amplitude of $\delta I$ is approximately $10^{-3}$. The top panel of Fig. 1 shows $\delta I$ measured at $20 \mathrm{TeV}$ with IceCube (Santander et al., 2013a). While only the southern sky is shown, a similar structure is consistently observed in the Northern Hemisphere by several experiments: a large-scale excess near $\alpha=110^{\circ}$, and a corresponding deficit near $\alpha=220^{\circ}$.

\subsection{Energy dependence}

The energy dependence of the large-scale anisotropy has been studied over a large energy range using the IceCube Neutrino Observatory. IceCube is comprised of 5160 optical modules deployed $1.4 \mathrm{~km}$ below the South Polar ice sheet, and it is sensitive to the $\mathrm{TeV}$ muons produced in cosmic ray air showers between $10 \mathrm{TeV}$ and $10 \mathrm{PeV}$. On the surface of 
the ice sheet, the IceTop detector of 86 surface stations is used to observe air showers above $100 \mathrm{TeV}$.

Figure 1 shows the large-scale structure observed by IceCube and IceTop (Santander et al., 2013a). The complete IceCube cosmic-ray data set, consisting of $>10^{11}$ individual air showers, has a median energy of $20 \mathrm{TeV}$, moderately higher in energy than the observations performed by surface arrays in the Northern Hemisphere. At $20 \mathrm{TeV}$ the anisotropy exhibits an excess near $\alpha=110^{\circ}$ and a deficit near $\alpha=220^{\circ}$.

Using the number of triggered optical modules and the zenith angle of the muons as a proxy estimate of the energy of the primary cosmic ray, the IceCube data can be used to produce a high-energy data set with a median energy of $400 \mathrm{TeV}$ (Abbasi et al., 2012). These data are shown in the middle panel of Fig. 1, and exhibit a "flip" in the orientation of the anisotropy, with a significant deficit present at $\alpha=110^{\circ}$. Because of the energy overlap between IceCube and IceTop above $100 \mathrm{TeV}$, it is possible to produce an independent intensity map of IceTop events with a median energy of $400 \mathrm{TeV}$ (Aartsen et al., 2013). The independent IceTop data set also exhibits a significant deficit at $\alpha=110^{\circ}$.

Due to the large dynamic range of IceTop, we may also produce a sky map of the cosmic rays with a median energy of $2 \mathrm{PeV}$; this map is shown in the bottom panel of Fig. 1 . At high energy the deficit at low right ascension persists, but its amplitude is nearly $3 \times 10^{-3}$, several times larger than at $400 \mathrm{TeV}$ (Aartsen et al., 2013).

The observation of large-scale structure can be explained as a consequence of the diffusion of cosmic rays from nearby sources in the galaxy. For example Erlykin and Wolfendale (2006), Blasi and Amato (2012), Pohl and Eichler (2013), and Sveshnikova et al. (2013) have conducted numerical experiments indicating that a large-scale anisotropy at the $10^{-3}$ level can easily arise from cosmic rays diffusing from nearby supernova remnants. Alternatively, Biermann et al. (2013) have proposed a magnetized field flow from old supernova remnants as the origin of the large-scale structures. While the anisotropy we observe is a particular realization of the distribution of sources in the galaxy, an average over many numerical simulations can produce the behavior observed in the data, including growth in the relative intensity as a function of energy and sudden flips in the phase of the anisotropy (Blasi and Amato, 2012; Pohl and Eichler, 2013; Sveshnikova et al., 2013).

\subsection{Time dependence}

Several authors have suggested that the heliosphere can have a significant influence on the cosmic-ray anisotropy (Desiati and Lazarian, 2013; Drury, 2013; Schwadron et al., 2014). Measurements of the time dependence of the anisotropy over periods comparable to one solar cycle may suggest the presence or absence of a heliospheric effect. In recent years conflicting measurements of time dependence have been reported in the literature. For example, the Milagro collabora-
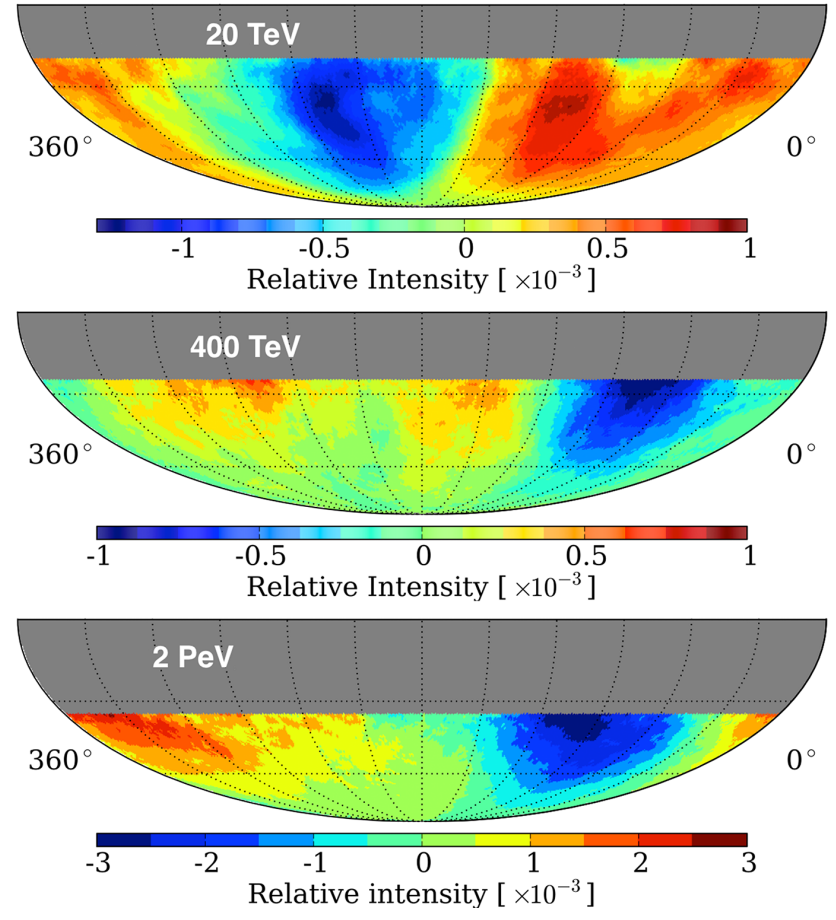

Figure 1. Top: large-scale anisotropy observed with IceCube, $20 \mathrm{TeV}$ median energy, from Santander et al. (2013a). Middle and bottom: IceCube $400 \mathrm{TeV}$ relative intensity map (Abbasi et al., 2012) and IceTop 2 PeV map (Aartsen et al., 2013).

tion claimed an increase in the amplitude of the dipole component of the anisotropy over seven years of measurements ending in 2007 (Abdo et al., 2009). In contrast, data from the Tibet $\mathrm{AS} \gamma$ detector indicated no significant changes in the anisotropy during nine years of measurements ending in 2008 (Amenomori et al., 2010).

More recently a long-term study of the cosmic-ray anisotropy has been reported by combining data from IceCube with its precursor experiment, the AMANDA detector (Santander et al., 2013a, b). The combined data cover twelve years of observation ending in 2012. No significant time variations in the large-scale anisotropy were observed during this period, with the exception of IceCube data from 2008 (Santander et al., 2013b). However, this was the first year of regular IceCube operations so it is possible that the variation is the result of an instability in the detector.

The AMANDA-IceCube data cover the second half of the 23rd solar cycle and the first half of the 24th solar cycle. IceCube will continue to collect cosmic ray data for the next decade, and the construction of new detectors such as HAWC will allow long-term simultaneous measurements of the anisotropy at complementary energy scales. 


\section{Small-scale anisotropy}

In addition to the large-scale anisotropy observed by many experiments since the year 2000, multiple experiments have also observed regions of excess and deficit on small angular scales of about $10^{\circ}$ to $20^{\circ}$. The small-scale structures were observed first by Milagro (Abdo et al., 2008), a water Cherenkov gamma-ray detector located in New Mexico, USA, and as fit residuals of the large-scale structure observed by the Tibet AS $\gamma$ array (Munakata et al., 2007). The Milagro Collaboration identifed two strong regions of excess at the $10^{-4}$ level: one located at $\alpha=60^{\circ}$, and a second located at $\alpha=120^{\circ}$. These two regions are shown in Fig. 2.

Though Milagro was a gamma-ray detector, a careful analysis of the data showed that these regions were comprised of hadronic cosmic rays. Monte Carlo simulations of several flux hypotheses indicated that the first region is described by a cutoff in the energy spectrum above $10 \mathrm{TeV}$, and the second region is described by a simple power law. The Milagro observations have been followed up and confirmed by the ARGO-YBJ Observatory (Bartoli et al., 2013) and the HAWC Observatory (BenZvi et al., 2013), which both have lower energy thresholds than Milagro. The relative intensity of the cosmic rays observed by HAWC is shown in Fig. 3 .

In the southern sky, IceCube data have been used to observe small-scale anisotropies beginning in 2008. The relative intensity observed by IceCube, determined for the complete data set with median energy $20 \mathrm{TeV}$, is shown in Fig. 2 (Santander et al., 2013a). Like the observations in the Northern Hemisphere, there is a significant excess at $\alpha=120^{\circ}$. However, the large excess at $\alpha=60^{\circ}$ is not present in the IceCube data. In addition, while the Northern Hemisphere measurements are dominated by excess regions, the southern sky shows regions of excess and deficit of equal amplitude.

The causes of the differences between the IceCube data and the observations in the Northern Hemisphere are not yet understood, but there are several possible explanations. One possibility is that the small-scale structure has a strong energy dependence, and so ARGO-YBJ and HAWC (1 TeV), Milagro $(1 \mathrm{TeV})$, and IceCube $(20 \mathrm{TeV})$ are not observing the same features. Another possibility is a difference in mass composition between the data. Above several $\mathrm{TeV}$ the flux of primary cosmic rays is dominated by helium over protons (Ahn et al., 2010). However, since IceCube detects cosmic rays by observing muons, it has a trigger bias against heavier nuclei at its energy threshold (Abbasi et al., 2011). This raises the possibility that IceCube is not observing a population of cosmic rays equivalent to that shown by lower-energy Northern Hemisphere experiments. The effect of mass composition on the anisotropy has not been studied, so this issue must be resolved with future analysis.

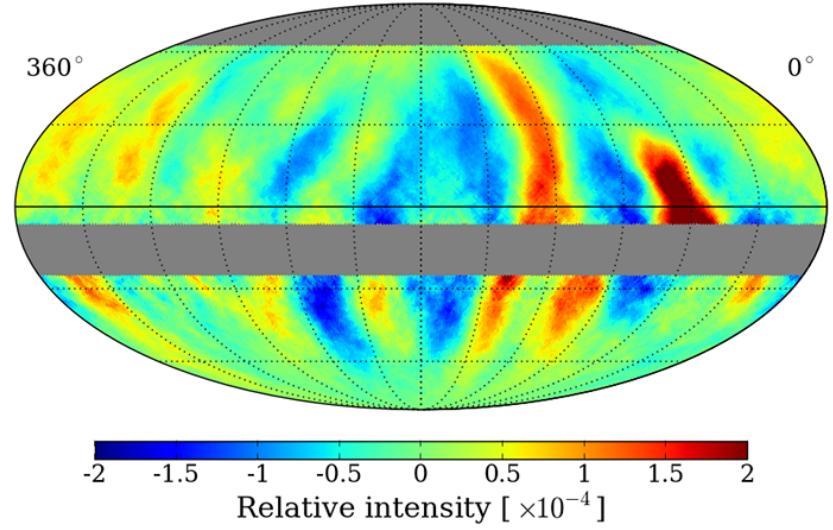

Figure 2. Small-scale anisotropy observed in the Northern Hemisphere by Milagro (Abdo et al., 2008) and in the Southern Hemisphere by IceCube (Santander et al., 2013a).

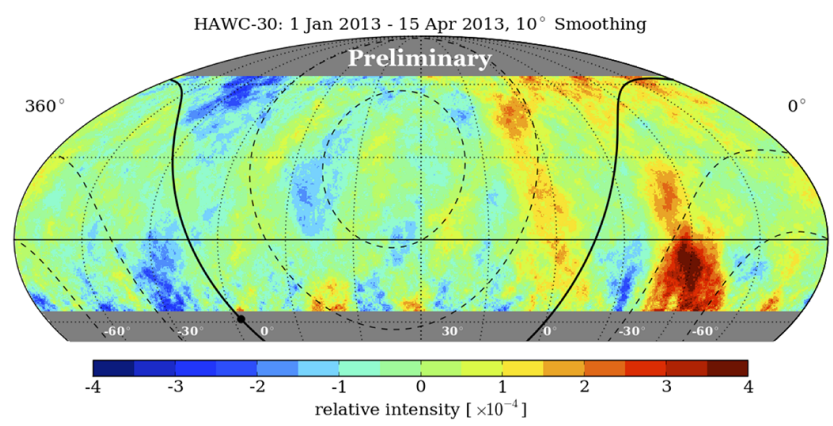

Figure 3. Small-scale anisotropy observed in the Northern Hemisphere by the HAWC Gamma-Ray Observatory (BenZvi et al., 2013).

\subsection{Time and energy dependence}

The time- and energy-dependence of the small-scale anisotropy has been investigated using IceCube and IceTop data (Abbasi et al., 2012; Aartsen et al., 2013). Unlike the large-scale structure, the small-scale anisotropy does not appear to persist to either the $400 \mathrm{TeV}$ or the $2 \mathrm{PeV}$ energy bands. However, there are no significant variations in the structures over time during the observation periods between 2008 and 2012.

\section{Origin of small-scale structure}

Following the initial observation of the small-scale anisotropy by Milagro, several authors hypothesized that unusual magnetic field configurations could allow concentrated beams of hadronic particles to propagate to Earth from accelerators hundreds of parsecs away (Drury and Aharonian, 2008; Salvati, 2010). 


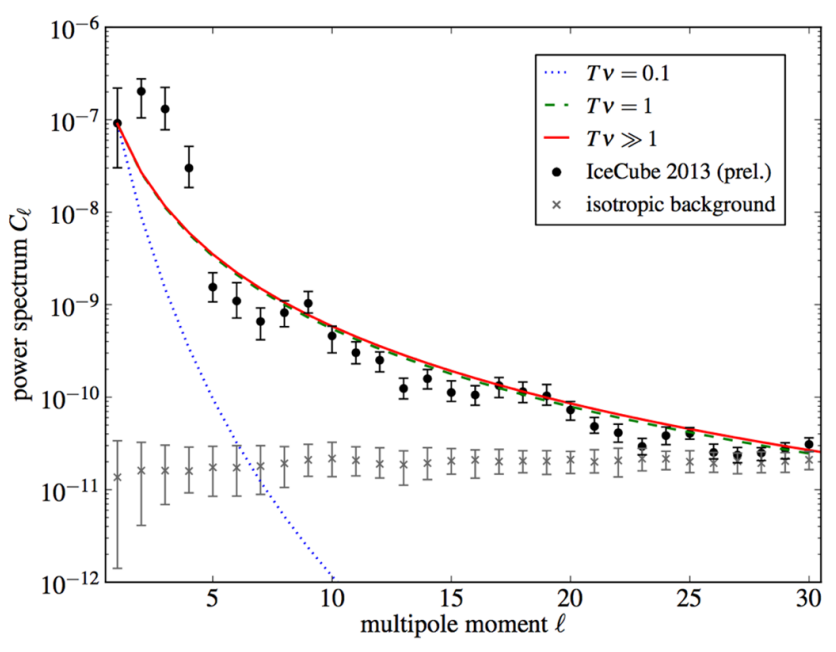

Figure 4. Comparison of the IceCube power spectrum against the angular correlations predicted by distortion of a large-scale anisotropy by a turbulent magnetic field. From Ahlers (2014).

More recent work has focused instead on the possibility that small-scale structure is produced by distortion of dipole anisotropies originating in particle diffusion as cosmic rays propagate through turbulent magnetic fields in the galaxy. For example, Giacinti and Sigl (2012) have carried out numerical simulations of cosmic ray diffusion in the galaxy to demonstrate that magnetic turbulence can give rise to $10^{\circ}$ to $20^{\circ}$ structures like those in real data. Of course, these studies are not predictive, as the actual structures observed at Earth are produced by the particular realization of the random magnetic field in our galaxy.

Ahlers (2014) has approached this same problem analytically, showing the effect of isotropic turbulence as a function of time on an initially dipolar anisotropy. The advantage of this technique is its quantative description of the distortion of a global dipole anisotropy into higher multipole anisotropies using the angular power spectrum of the data.

Figure 4 shows the angular power spectrum of the combined IceCube cosmic-ray arrival direction distribution recorded between 2008 and 2012 (Santander et al., 2013a; Ahlers, 2014). The data points are helpful to visualize the relatively large amount of power at low multipole moments $\ell<5$ (see Figs. 1 and 2), as well as the small but still significant power at moments up to $\ell=20$, which correspond to angular scales of order $10^{\circ}$. The light gray band indicates the expected power spectrum of an isotropic angular distribution of cosmic rays, estimated for many random realizations of the IceCube data.

According to the model developed by Ahlers (2014), the sum of multipoles in the power spectrum is conserved while high-order multipoles grow (in relative terms) as cosmic rays propagate through a turbulent field. As a result, an initially dipolar distribution will leak into higher multipole moments. The asymptotic strength of the high-order multipoles at times much longer than the diffusion relaxation time is shown as a red curve in Fig. 4. This curve appears to be a reasonable description of the IceCube data above $\ell=5$, supporting the hypothesis that the small-scale anisotropy originates in magnetic scattering.

\section{Conclusions}

A $10^{-3}$ anisotropy in the TeV-PeV cosmic rays has been established with a full decade of observations in both the Northern and Southern Hemispheres. The energy dependence of the anisotropy exhibits a change in relative orientation and an increase in amplitude as a function of energy, which are expected features of the diffusion of cosmic rays from nearby galactic accelerators. Simulations of diffusion also indicate that turbulent magnetic fields can explain the origin of the small-scale anisotropy. This hypothesis is further supported by the shape of the angular power spectrum.

At low energies $(<10 \mathrm{TeV})$, conflicting measurements have been reported on the time dependence of the anisotropy. However, above $10 \mathrm{TeV}$ both the large- and small-scale structures appear to be stable over times comparable to the length of the solar cycle. With IceCube and HAWC expected to record very large numbers of cosmic rays during the next decade, we should soon have significant data to study the influence of the heliosphere on the anisotropy.

Acknowledgements. This work has been supported by the U.S. National Science Foundation - Physics Division. The author would like to thank M. Santander, M. Ahlers, A. Kappes, and J. Tjus for helpful suggestions.

Edited by: J. Tjus

Reviewed by: two anonymous referees

\section{References}

Aartsen, M., Abasi, R., Abdou, Y., et al.: Observation of CosmicRay Anisotropy with the IceTop Air Shower Array, Astrophys. J., 765, 55, doi:10.1088/0004-637X/765/1/55, 2013.

Abbasi, R., Abdou, Y., Abu-Zayyad, T., et al.: Measurement of the Anisotropy of Cosmic-ray Arrival Directions with IceCube, Astrophys. J., 718, L194, doi:10.1088/2041-8205/718/2/L194, 2010.

Abbasi, R., Abdou, Y., Abu-Zayyad, T., et al.: Observation of Anisotropy in the Arrival Directions of Galactic Cosmic Rays at Multiple Angular Scales with IceCube, Astrophys. J., 740, 16, doi:10.1088/0004-637X/740/1/16, 2011.

Abbasi, R., Abdou, Y., Abu-Zayyad, T., et al.: Observation of Anisotropy in the Galactic Cosmic-Ray Arrival Directions at $400 \mathrm{TeV}$ with IceCube, Astrophys. J., 746, 33, doi:10.1088/0004637X/746/1/33, 2012.

Abdo, A. A., Allen, B., Aune, T., Berley, D., Blaufuss, E., Casanova, S., Chen, C., Dingus, B. L., Ellsworth, R. W., Fleysher, L., Fleysher, R., Gonzales, M. M., Goodman, J. A., Hoffman, C. M., Hüntemeyer, P. H., Kolterman, B. E., Lansdell, C. P., Linnemann, 
J. T., McEnery, J. E., Mincer, A. I., Nemethy, P., Noyes, D., Pretz, J., Ryan, J. M., Saz Parkinson, P. M., Shoup, A., Sinnis, G., Smith, A. J., Sullivan, G. W., Vasileiou, V., Walker, G. P., Williams, D. A., and Yodh, G. B.: Discovery of Localized Regions of Excess 10-TeV Cosmic Rays, Phys. Rev. Lett., 101, 221101, doi:10.1103/PhysRevLett.101.221101, 2008.

Abdo, A. A., Allen, B. T., Aune, T., Berley, D., Casanova, S., Chen, C., Dingus, B. L., Ellsworth, R. W., Fleysher, L., Fleysher, R., Gonzalez, M. M., Goodman, J. A., Hoffman, C. M., Hopper, B., Hüntemeyer, P. H., Kolterman, B. E., Lansdell, C. P., Linnemann, J. T., McEnery, J. E., Mincer, A. I., Nemethy, P., Noyes, D., Pretz, J., Ryan, J. M., Saz Parkinson, P. M., Shoup, A., Sinnis, G., Smith, A. J., Sullivan, G. W., Vasileiou, V., Walker, G. P., Williams, D. A., and Yodh, G. B.: The Large-Scale Cosmic-Ray Anisotropy as Observed with Milagro, Astrophys. J., 698, 21212130, doi:10.1088/0004-637X/698/2/2121, 2009.

Aglietta, M., Alekseenko, V. V., Alessandro, B., Antonioli, P., Arneodo, F., Bergamasco, L., Bertaina, M., Bonino, R., Castellina, A., Chiavassa, A., D’Ettorre Piazzoli, B., Di Sciascio, G., Fulgione, W., Galeotti, P., Ghia, P. L., Iacovacci, M., Mannocchi, G., Morello, C., Navarra, G., Saavedra, O., Stamerra, A., Trinchero, G. C., Valchierotti, S., Vallania, P., Vernetto, S., and Vigorito, C. (The EAS-TOP Collaboration): Evolution of the cosmic ray anisotropy above $10^{14} \mathrm{eV}$, Astrophys. J. Lett., 692, L130-L133, doi:10.1088/0004-637X/692/2/L130, 2009.

Ahlers, M.: Anomalous Anisotropies of Cosmic Rays from Turbulent Magnetic Fields, Phys. Rev. Lett., 112, 021101, doi:10.1103/PhysRevLett.112.021101, 2014.

Ahn, H., Allison, P., Bagliesi, M. G., Beatty, J. J., Bigongiari, G., Childers, J. T., Conklin, N. B., Coutu, S., DuVernois, M. A., Ganel, O., Han, J. H., Jeon, J. A., Kim, K. C., Lee, M. H., Lutz, L., Maestro, P., Malinin, A., Marrocchesi, P. S., Minnick, S., Mognet, S. I., Nam, J., Nam, S., Nutter, S. L., Park, I. H., Park, N. H., Seo, E. S., Sina, R., Wu, J., Yang, J., Yoon, Y. S., Zei, R., and Zinn, S. Y.: Discrepant hardening observed in cosmic-ray elemental spectra, Astrophys. J., 714, L89-L93, doi:10.1088/2041-8205/714/1/L89, 2010.

Amenomori, M., Ayabe, S., Cui, S. W., et al.: Large-Scale Sidereal Anisotropy of Galactic Cosmic-Ray Intensity Observed by the Tibet Air Shower Array, Astrophys. J., 626, L29-L32, doi:10.1086/431582, 2005.

Amenomori, M., Bi, X. J., Chen, D., et al.: On Temporal Variations of the Multi-TeV Cosmic Ray Anisotropy Using the Tibet III Air Shower Array, Astrophys. J., 711, 119-124, doi:10.1088/0004637X/711/1/119, 2010.

Bartoli, B., Bernardini, P., Bi, X. J., et al.: Medium scale anisotropy in the $\mathrm{TeV}$ cosmic ray flux observed by ARGO-YBJ, Phys. Rev. D, 88, 082001, doi:10.1103/PhysRevD.88.082001, 2013.

BenZvi, S. Y. et al.: Observations of the Anisotropy of Cosmic Rays with HAWC, in: Proc. 33rd ICRC, Rio de Janeiro, Brazil, 2013.

Biermann, P., Becker Tjus, J., Seo, E.-S., and Mandelartz, M.: Cosmic-Ray Transport and Anisotropies, Astrophys. J., 768, 124, doi:10.1088/0004-637X/768/2/124, 2013.
Blasi, P. and Amato, E.: ffusive propagation of cosmic rays from supernova remnants in the Galaxy. II: anisotropy, J. Cosmol. Astropart. P., 1201, 011, doi:10.1088/1475-7516/2012/01/011, 2012.

de Jong, J.: Observations of Large Scale Sidereal Anisotropy in 1 and $11 \mathrm{TeV}$ cosmic rays from the MINOS experiment, in: Proc. 32nd ICRC, Beijing, China, 2011.

Desiati, P. and Lazarian, A.: Anisotropy of TeV Cosmic Rays and Outer Heliospheric Boundaries, Astrophys. J., 762, 44, doi:10.1088/0004-637X/762/1/44, 2013.

Di Sciascio, G.: Measurement of Cosmic Ray Spectrum and Anisotropy with ARGO-YBJ, EPJ Web of Conferences 52, 04004, doi:10.1051/epjconf/20125204004, 2013.

Drury, L.: The problem of small angular scale structure in the cosmic ray anisotropy data, in: Proc. 33rd ICRC, Rio de Janeiro, Brazil, 2013.

Drury, L. and Aharonian, F.: The puzzling MILAGRO hot spots, Astropart. Phys., 29, 420-423, 2008.

Erlykin, A. D. and Wolfendale, A.: The anisotropy of galactic cosmic rays as a product of stochastic supernova explosions, Astropart. Phys., 25, 183-194, 2006.

Giacinti, G. and Sigl, G.: Local Magnetic Turbulence and TeVPeV Cosmic Ray Anisotropies, Phys. Rev. Lett., 109, 071101, doi:10.1103/PhysRevLett.109.071101, 2012.

Guillian, G., Hosaka, J., Ishihara, K., et al.: Observation of the anisotropy of $10 \mathrm{TeV}$ primary cosmic ray nuclei flux with the Super-Kamiokande-I detector, Phys. Rev. D, 75, 062003, doi:10.1103/PhysRevD.75.062003, 2007.

Munakata, K., et al.: Implication of the sidereal anisotropy of $5 \mathrm{TeV}$ cosmic ray intensity observed with the Tibet III air shower array, in: Proc. 30th ICRC, Mérida, México, 2007.

Pohl, M. and Eichler, D.: Understanding TeV-band CosmicRay Anisotropy, Astrophys. J., 766, 4, doi:10.1088/0004637X/766/1/4, 2013.

Salvati, M.: The local Galactic magnetic field in the direction of Geminga, Astron. Astrophys., 513, A28, doi:10.1051/00046361/200913406, 2010.

Santander, M., Desiati, P., BenZvi, S., and Westerhoff, S.: Observations of the Anisotropy of Cosmic Rays with HAWC, in: Proc. 33rd ICRC, Rio de Janeiro, Brazil, $2013 \mathrm{a}$.

Santander, M., Desiati, P., Gurtner, M., Kampert, K.-H., et al.: Observations of the Anisotropy of Cosmic Rays with HAWC, in: Proc. 33rd ICRC, Rio de Janeiro, Brazil, 2013b.

Schwadron, N. A., Adams, F. C., Christian, E. R., Desiati, P., Frisch, P., Funsten, H. O., Jokipii, J. R., McComas, D. J., Moebius, E., and Zank, G. P.: Global Anisotropies in TeV Cosmic Rays Related to the Sun's Local Galactic Environment from IBEX, Science, 343, 988-990, doi:10.1126/science.1245026, 2014.

Sveshnikova, L., Strelnikova, O. N., and Ptuskin, V. S.: Spectrum and Anisotropy of Cosmic Rays at TeV-PeV-energies and Contribution of Nearby Sources, Astropart. Phys., 50-52, 33-46, doi:10.1016/j.astropartphys.2013.08.007, 2013. 